\title{
Knockdown of $\mathrm{Mg}^{2+} \mathrm{Mn}^{2+}$ dependent protein phosphatase 1A promotes apoptosis in BV2 cells infected with Brucella suis strain 2 vaccine
}

\author{
JUAN YANG $^{1 *}$, GUOWEI WANG $^{1 *}$, HAINING LI $^{1 *}$, WENLI ZHENG ${ }^{1}$, BURUI GUO $^{1}$ and ZHENHAI WANG ${ }^{1,2}$ \\ ${ }^{1}$ Department of Neurology, The General Hospital of Ningxia Medical University; \\ ${ }^{2}$ Ningxia Key Laboratory of Cerebrocranial Diseases, Incubation Base of National Key Laboratory, \\ Yinchuan, Ningxia 750004, P.R. China
}

Received January 16, 2019; Accepted December 10, 2019

DOI: $10.3892 /$ etm.2020.8745

\begin{abstract}
The ability to inhibit host macrophage apoptosis is one of the survival strategies of intracellular bacteria, including Brucella. In the present study the role of $\mathrm{Mg}^{2+/} \mathrm{Mn}^{2+}$ dependent protein phosphatase 1A (PPM1A) in the apoptosis of Brucella suis (B. suis) strain 2 vaccine-infected BV2 cells was investigated. Compared with control cells, the protein expression levels of cleaved caspase-3 were markedly increased in PPM1A short hairpin (sh)RNA-transfected BV2 cells. Flow cytometry analysis showed that treatment with JNK activator anisomycin significantly increased the rate of apoptosis in BV2 cells in comparison with the control cells. Furthermore, PPM1A shRNA significantly increased the levels of JNK phosphorylation and the levels of cleaved caspase-3 in BV2 cells infected with B. suis strain 2 in comparison with the control cells. DAPI staining showed nuclear condensation in B. suis infected BV2 cells transfected with PPM1A shRNA in comparison with the control shRNA cells. Flow cytometry analysis showed that PPM1A shRNA significantly increased the percentage of apoptotic BV2 cells infected with B. suis strain 2 compared with those transfected with control shRNA. Taken together, these data suggested that knockdown of PPM1A promotes apoptosis in B. suis strain 2-infected BV2 cells and that PPM1A may be a potential target in the development of treatments to inhibit intracellular growth of B. suis.
\end{abstract}

Correspondence to: Dr Zhenhai Wang, Department of Neurology, The General Hospital of Ningxia Medical University, 804 South Shengli Street, Yinchuan, Ningxia 750004, P.R. China

E-mail: wangzhenhai1968@163.com

*Contributed equally

Key words: $\mathrm{Mg}^{2+} \mathrm{Mn}^{2+}$ dependent protein phosphatase 1A, JNK, apoptosis, Brucella suis vaccine strain 2, microglia, BV2 cells

\section{Introduction}

Brucellosis is a zoonotic infectious disease caused by infection with the bacterial genus Brucella. Brucella are able to localize to phagocytic cells in human organs including the liver, spleen, bone marrow and brain, thereby leading to various clinical manifestations (1). Brucella suis (B. suis) can cause devastating multi-organ diseases in humans, which lead to severe health complications (2-4). Previous studies have shown that Brucella has several strategies to establish chronic infection, including inhibition of apoptosis in infected mononuclear cells, inhibition of dendritic cell maturation, and a reduction in the antigen presentation ability and activation of naïve T-cells $(5,6)$. One possible strategy for treatment of $B$. suis infection is the promotion of apoptosis in host cells. $B$. suis strain 2 is a low virulence live-strain vaccine known to improve cellular immunity and protect animals against infection by heterologous virulent Brucella (7). The properties of B. suis strain 2 make it a suitable model for in vitro study of Brucella.

$\mathrm{Mg}^{2+} / \mathrm{Mn}^{2+}$ dependent protein phosphatase 1A (PPM1A), a member of the serine/threonine phosphatase family, is known to be a critical regulator of cellular apoptosis (8). It has been shown that PPM1A is a key factor in the innate antibacterial and antiviral response of macrophages, particularly in Mycobacterium tuberculosis infection $(9,10)$. As both Brucella and Mycobacterium tuberculosis effectively function as intracellular parasites, sharing similarities in their pathogenesis $(11,12)$ it is hypothesized in the present study that PPM1A may also regulate apoptosis in B. suis infection.

Microglia, resident immune cells in the brain, are involved in normal brain development and neuronal recovery (13). It has been reported that Brucella infection activates microglia and leads to neuronal loss, thereby contributing to neurological deficits observed during neurobrucellosis. In the present study, the role of PPM1A in the regulation of apoptosis was investigated in BV2 cells, an immortalized mouse cell line that models microglia, that had been infected with $B$. suis strain 2 . 


\section{Materials and methods}

Cell lines and bacteria. Mouse microglia cell line BV2 cells were provided by American Type Culture Collection and cultured in DMEM (Thermo Fisher Scientific Inc.) containing $10 \%$ FBS (Hyclone; GE Healthcare Life Sciences), $2 \mathrm{mM}$ glutamine and $200 \mathrm{mM}$ streptomycin/penicillin (Beijing Solarbio Science and Technology Co., Ltd.) and maintained in $5 \% \mathrm{CO}_{2}$ at $37^{\circ} \mathrm{C}$. B. suis strain 2 was a kind gift from Professor $\mathrm{Xu}$ of Ningxia Medical University (Yinchuan, China) and was cultured on trypticase soy-agar plates at $37^{\circ} \mathrm{C}$ in a $5 \% \mathrm{CO}_{2}$ incubator. Individual B. suis strain 2 colonies were seeded in sterilized trypticase soy broth solution at $37^{\circ} \mathrm{C}$ in $5 \% \mathrm{CO}_{2}$. Bacteria were harvested by centrifugation for $20 \mathrm{~min}$ at $2,000 \mathrm{~g}$ at $4^{\circ} \mathrm{C}$ and washed twice with PBS. Bacterial density in the culture was estimated using a McFarland standards kit (bioMérieux China Ltd.). All experiments involving bacteria were performed in a biosafety level 2 laboratory.

In vitro infection. BV2 cells $\left(8 \times 10^{5}\right)$ were grown in six-well cell culture plates, allowed to reach $60 \%$ confluence, and then exposed to B. suis strain 2 at multiplicity of infection (MOI) 100 for $1 \mathrm{~h}$ in DMEM without antibiotics. Thereafter, BV2 cells were washed extensively, to remove extracellular bacteria. The infection was maintained for $24 \mathrm{~h}$ in the presence of $100 \mu \mathrm{g} / \mathrm{ml}$ gentamicin, to kill any remaining extracellular bacteria. Medium and cells were collected for subsequent experiments. BV2 cells were infected at different intervals $(0,4,8$ and $24 \mathrm{~h})$ for western blotting and at 24 for the remaining analysis. In addition, BV2 cells were also infected in shRNA experiments.

Cell viability assay. Cell viability was determined using a Cell Counting Kit-8 (CCK-8) assay according to the manufacturer's protocol (Nanjing Fengfeng Biomedical Technology Co., Ltd.). In brief, uninfected BV2 cells were seeded in 96-well cell culture plates at a density of $1 \times 10^{4}$ cells $/ \mathrm{ml}$ and cultured overnight at $37^{\circ} \mathrm{C}$. Cells were treated with SP600125 (MedChemExpress; 5 and $10 \mu \mathrm{M}$ ) and anisomycin (MedChem Express; 0.2,0.5 and $1 \mu \mathrm{M}$ ) for $24 \mathrm{~h}$. Cells treated with DMSO served as controls. Subsequently, CCK- 8 solution was added to each well and incubated for an additional $4 \mathrm{~h}$. The absorbance at $450 \mathrm{~nm}$ was measured using a microplate reader (BioTek Instruments, Inc.).

Short hairpin (sh)RNA expression constructs and virus infection. Lentiviral vector GV493 and an shRNA plasmid coding for PPM1A were purchased from Shanghai Genechem Co., Ltd. The shRNA targeting PPM1A had the sequence 5'-GAGAGT TATGTCAGAGAAGAA-3'. The scrambled RNA sequence, used as a control, had the sequence 5'-TTCTCCGAACGTGTC ACGT-3'. BV2 cells were infected with viruses expressing control shRNA or shRNA targeting PPM1A at MOI 50. BV2 cells were used $72 \mathrm{~h}$ after transfection, and stable cell lines were established as previously described and were selected for 5 days using puromycin (Sigma-Aldrich; Merck KGaA; $2 \mu \mathrm{g} / \mathrm{ml})(14)$.

Flow cytometry. BV2 cells ( $1 \times 10^{5}$ cells) were treated with $5 \mu \mathrm{M}$ SP600125 or $0.2 \mu \mathrm{M}$ anisomycin at $37^{\circ} \mathrm{C}$ for $48 \mathrm{~h}$ and stained with annexin $\mathrm{V}$ conjugated to FITC or propidium iodide

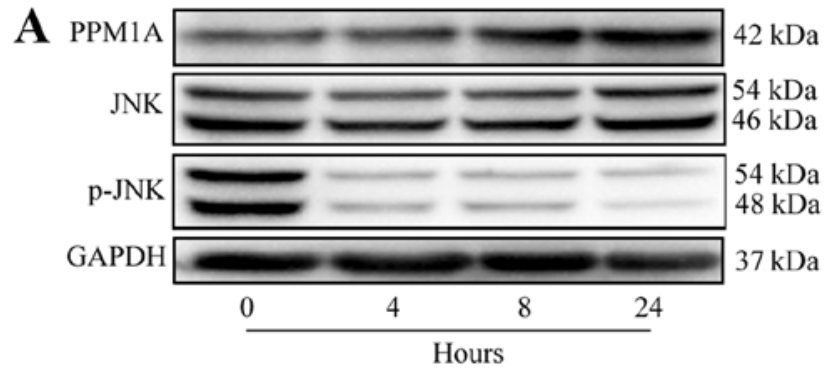

B
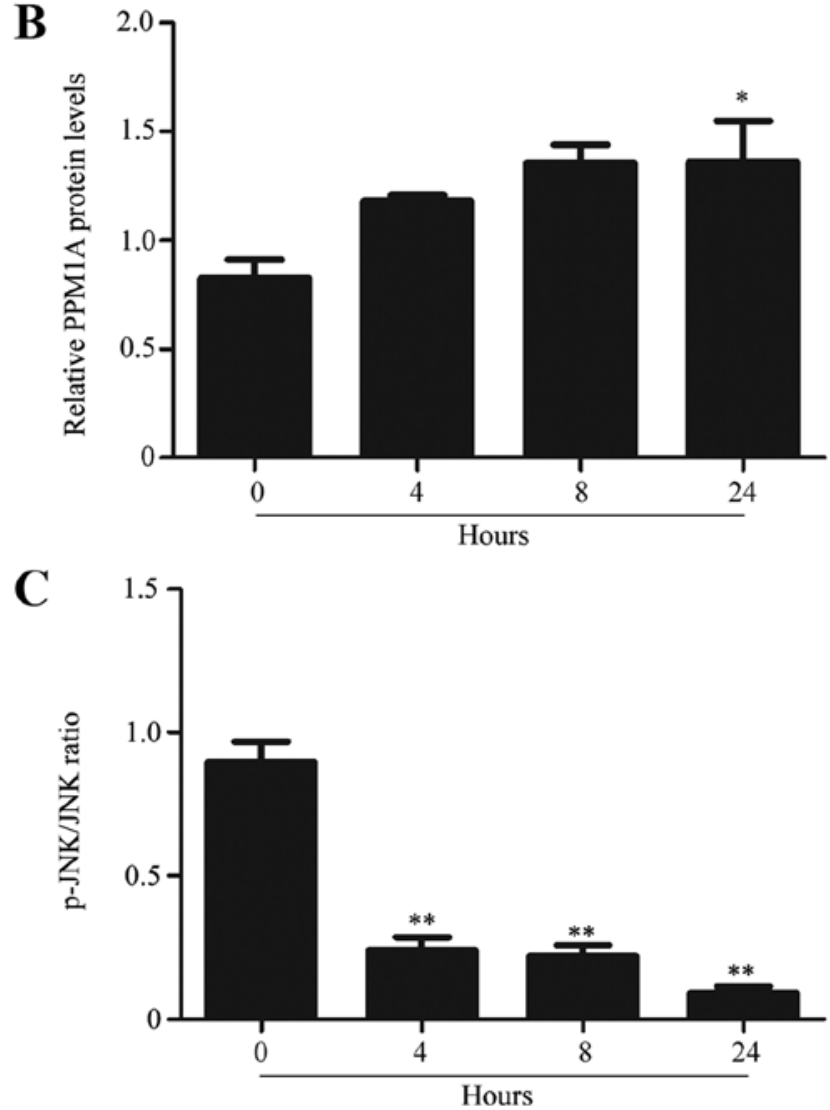

Figure 1. Expression of PPM1A and reduced phosphorylation of JNK in Brucella suis strain 2-infected BV2 cells. (A) Representative blots from three independent experiments. Quantification of (B) PPM1A and (C) p-JNK protein levels from three separate experiments, normalized to GAPDH. ${ }^{*} \mathrm{P}<0.05$, ${ }^{* *} \mathrm{P}<0.01$ vs. 0 h. p, phosphorylated; PPM1A, $\mathrm{Mg}^{2+/} \mathrm{Mn}^{2+}$ dependent protein phosphatase $1 \mathrm{~A}$.

according to the manufacturer's protocol (cat. no. BB-4101-1; Nanjing Fengfeng Biomedical Technology Co.,Ltd.). Apoptosis was determined by flow cytometry using a BD Accuri C6. Data analysis was performed using BD Accuri $\mathrm{C}^{\mathrm{TM}} 6$ software Plus (BD Biosciences).

Western blot analysis. BV2 cells were harvested, washed twice with PBS, and lysed in RIPA buffer (Nanjing Jiancheng Bioengineering Institute). Protein concentrations in the lysates were quantified by the bicinchoninic acid method following the manufacturer's instructions (Nanjing KeyGen Biotech Co., Ltd.). A total of $30 \mu \mathrm{g}$ of protein per sample was loaded and separated on $12 \%$ Mini-Protean ${ }^{\circledR}$ TGX $^{\mathrm{TM}}$ gels (Bio-Rad Laboratories, Inc.) and subsequently transferred onto a polyvinylidene difluoride membrane. Prior to antibody incubations, the samples were blocked 
A
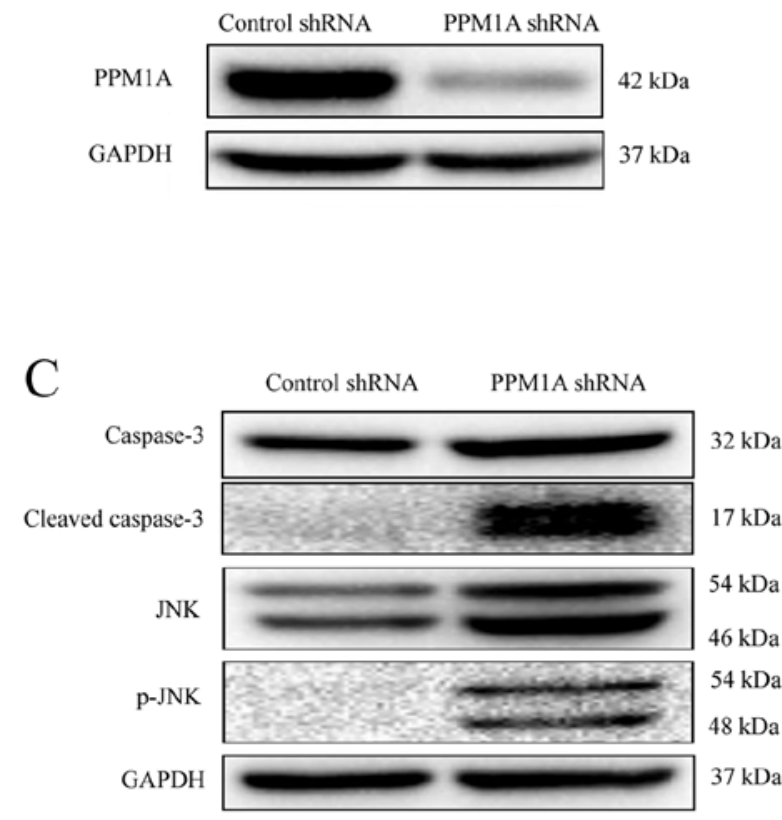

E

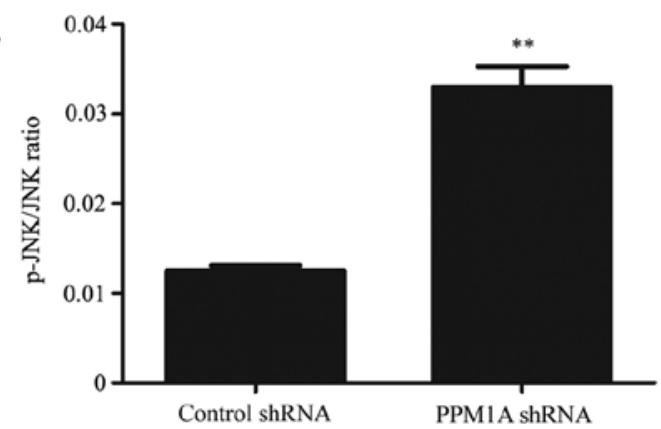

B

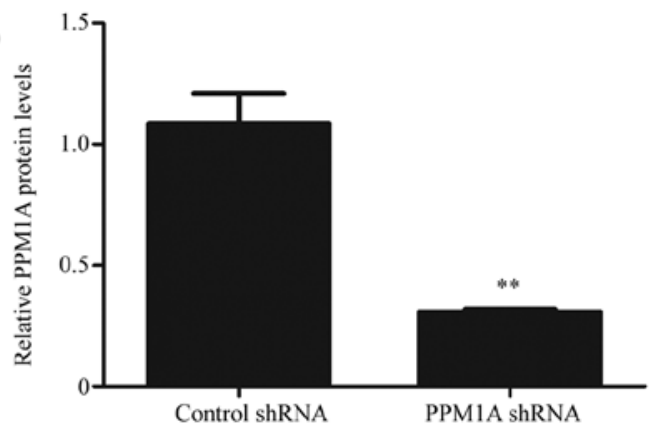

D

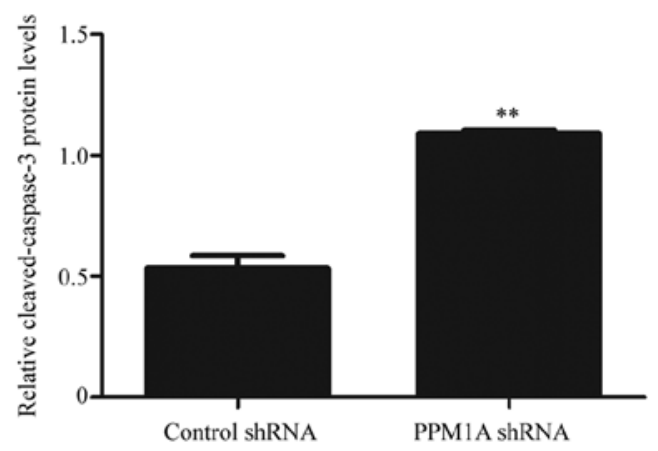

Figure 2. Knockdown of PPM1A by transfection of shRNA in BV2 cells. (A) BV2 cells were transfected with PPM1A shRNA and control shRNA, and western blot analysis was performed to determine the protein levels of PPM1A. (B) Densitometry of PPM1A protein levels. (C) Western blot analysis was also performed to assess the levels of caspase-3, cleaved caspase-3, JNK and p-JNK. The blots shown are representative of three independent experiments. Densitometric quantification data is expressed as the intensity ratio of target proteins (D) cleaved caspase-3 and (E) p-JNK to GAPDH. * P $<0.01$ vs. control shRNA cells. PPM1A, $\mathrm{Mg}^{2+} \mathrm{Mn}^{2+}$ dependent protein phosphatase 1A; p, phosphorylated; shRNA, short hairpin RNA.

with $5 \%$ skimmed milk at room temperature for $1 \mathrm{~h}$. PPM1A (dilution 1:1,000; cat. no. ab14824), JNK (dilution 1:1,000; cat. no. ab179461), phosphorylated (p)-JNK (dilution 1:3,000; cat. no. ab4821), caspase-3 (dilution 1:1,000; cat. no. ab13847), cleaved caspase-3 (dilution 1:500; cat. no. ab2302) and GAPDH (dilution 1:1,000; cat. no. ab181602) protein levels were assessed using specific antibodies (Abcam) at $4^{\circ} \mathrm{C}$ overnight. Horseradish peroxidase-conjugated goat anti-mouse polyclonal antibody (dilution 1:5,000; Santa Cruz Biotechnology, Inc.; cat. no. sc-2031) was used as the secondary antibody for $30 \mathrm{~min}$ at room temperature. The blot was developed using Western Lightning Ultra chemiluminescent substrate (Bio-Rad Laboratories, Inc.) in an EpiChemi3 darkroom (UVP, LLC). Image Lab 3.0 software used to analyze the results (Bio-Rad Laboratories, Inc.).

DAPI staining. BV2 cells were seeded on six-well plates at a density of $5 \times 10^{5}$ cells $/ \mathrm{ml}$ and cultured overnight at $37^{\circ} \mathrm{C}$. Cells were fixed in $4 \%$ paraformaldehyde at room temperature for
$1 \mathrm{~h}$. After washing with PBS and air-drying for $3 \mathrm{~min}$ at room temperature, cells were stained with DAPI at room temperature for $1 \mathrm{~min}$ and images were captured using fluorescence microscopy immediately (x200 magnification, 6 random field were viewed).

Statistical analysis. All data are presented as the mean \pm SD from at least three independent experiments. Graphpad Prism 7.0 (GraphPad Software, Inc.) was used for the analysis. Comparisons between indicated groups were performed using one-way analysis of variance followed by Tukey's post-hoc test. $\mathrm{P}<0.05$ was considered to indicate a statistically significant difference.

\section{Results}

Increased expression of PPM1A and reduced phosphorylation of JNK are observed in B. suis strain 2-infected BV2 cells. The expression of PPM1A and JNK proteins was evaluated by western blot analysis in BV2 cells at 4,8 and 24 h post- $B$. suis 
A

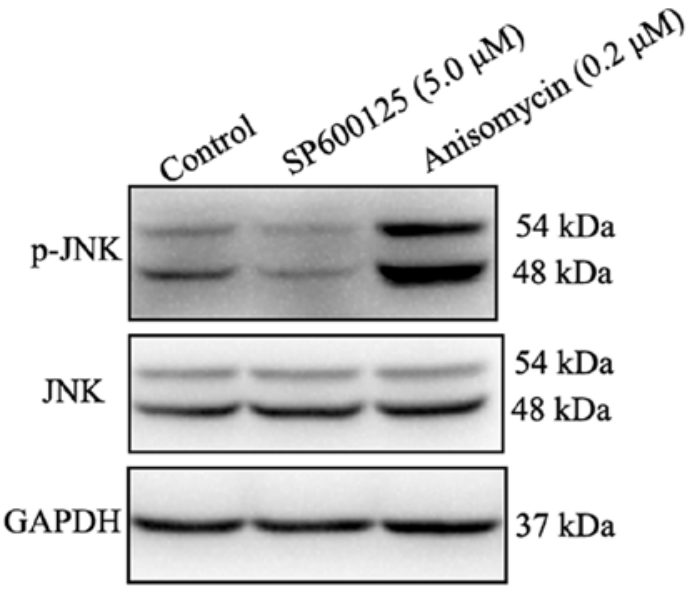

B

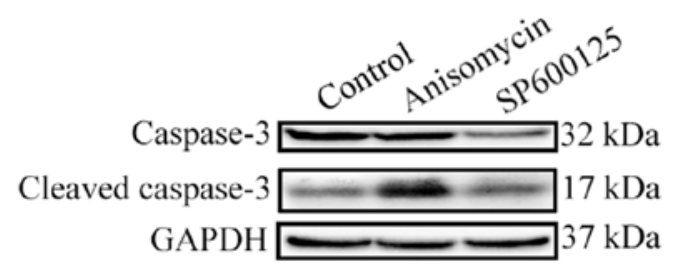

C

Control

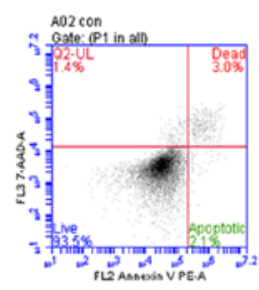

SP600125

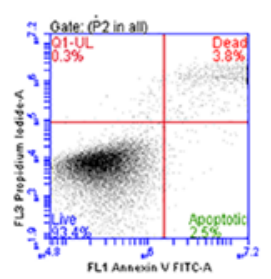

Anisomycin

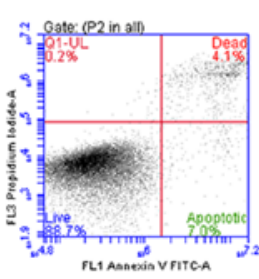

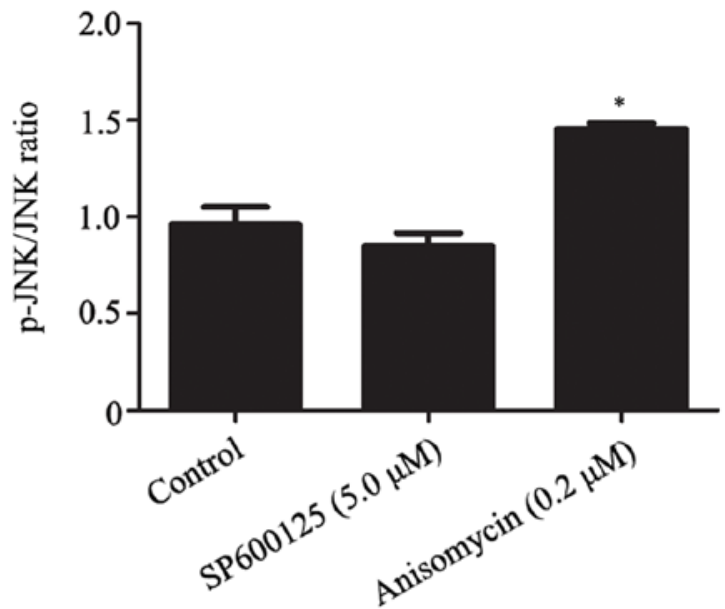
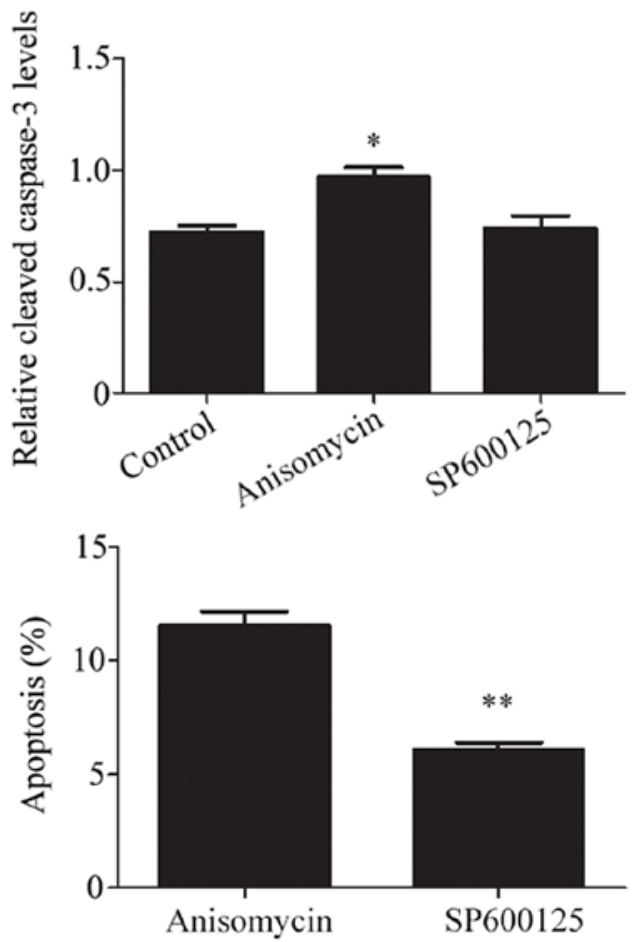

Figure 3. Effects of JNK modulation by SP100625 and anisomycin on apoptosis in BV2 cells. BV2 cells were treated with indicated concentrations of SP100625 or anisomycin, and western blot analysis was used to evaluate the expression of (A) p-JNK and JNK and (B) caspase-3 and cleaved caspase-3. (C) BV2 cells were treated with SP600125 at $5 \mu \mathrm{M}$ or anisomycin at $0.2 \mu \mathrm{M}$, and stained with Annexin V conjugated to FITC and propidium iodide. The percentage of cells undergoing apoptosis is shown. The total number of apoptotic cells was estimated from the sum of cells in early apoptosis (lower right) and late apoptosis (upper right). ${ }^{*} \mathrm{P}<0.05,{ }^{* *} \mathrm{P}<0.01$ vs. control cells. p, phosphorylated.

strain 2 infection. The results indicated that PPM1 A expression was significantly increased and phosphorylation of JNK was significantly reduced in a time-dependent manner compared with control shRNA-infected cells (Fig. 1).

PPMIA knockdown promotes caspase-dependent apoptosis in uninfected BV2 cells. To further characterize the role of PPM1A in apoptosis regulation, RNA interference was used to knock down PPM1A expression in BV2 cells. The results indicated that PPM1A protein levels in cells transfected with shRNA specific for PPM1A were significantly reduced compared with those transfected with control shRNA (Fig. 2A and B). This suggested that shRNA targeting
PPM1A mRNA could effectively knockdown PPM1A expression at a translational level. Compared with control shRNA cells, the protein expression levels of cleaved caspase- 3 were markedly increased in PPM1A shRNA-transfected BV2 cells (Fig. 2C and D). Collectively, these results suggested that knockdown of PPM1A promoted caspase-dependent apoptosis in BV2 cells.

Activation of JNK signaling promotes apoptosis in BV2 cells. To determine whether JNK signaling was involved in apoptosis, uninfected BV2 cells were treated with the JNK inhibitor SP100625 or JNK activator anisomycin. The CCK-8 assay showed that treatment with SP600125 at $5 \mu \mathrm{M}$ or aniso- 

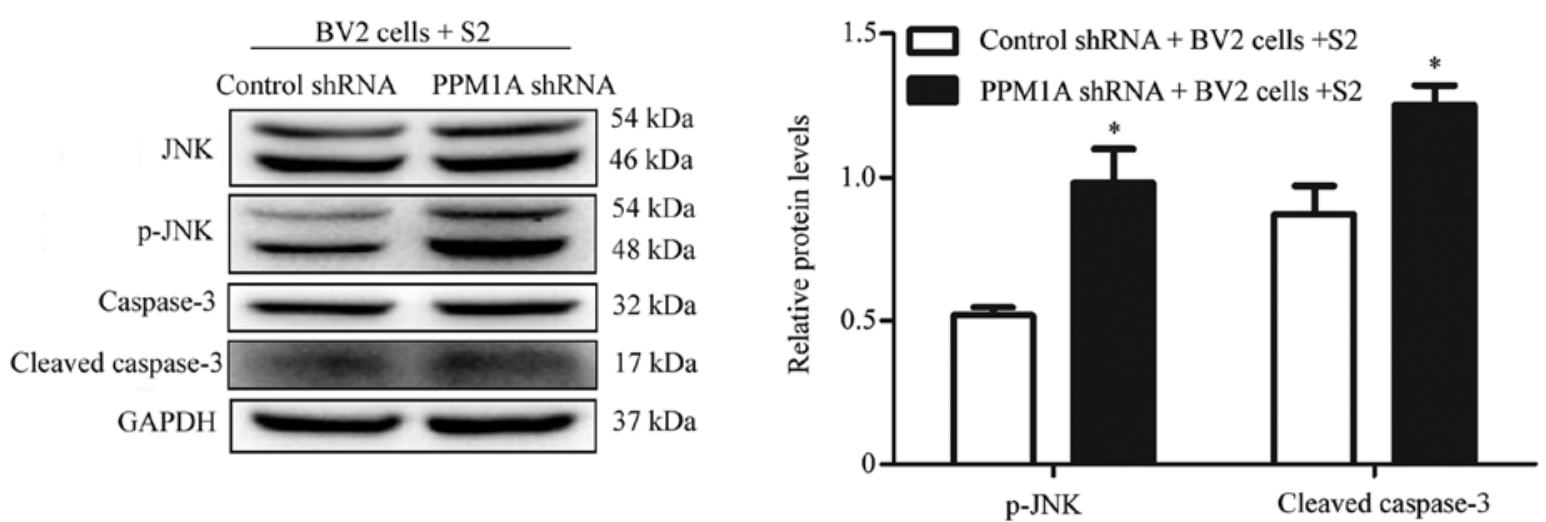

Figure 4. Effects of PPM1A knockdown on protein levels of JNK, p-JNK, caspase-3 and cleaved caspase-3 in BV2 cells infected with B. suis s2. BV2 cells infected with $B$. suis s2 were transfected with PPM1A shRNA or control shRNA. Cells were collected for western blot analysis. The blots shown are representative of three independent experiments. Densitometric quantification data are expressed as the intensity ratio of target proteins to total JNK or GAPDH. ${ }^{*} \mathrm{P}<0.05$ vs. control shRNA + BV2 cells + s2. B. suis, Brucella suis; PPM1A, $\mathrm{Mg}^{2+} \mathrm{Mn}^{2+}$ dependent protein phosphatase 1A; p, phosphorylated; s2, strain 2; shRNA, short hairpin RNA.

A

$\mathrm{BV} 2$ cells $+\mathrm{S} 2$

Control shRNA

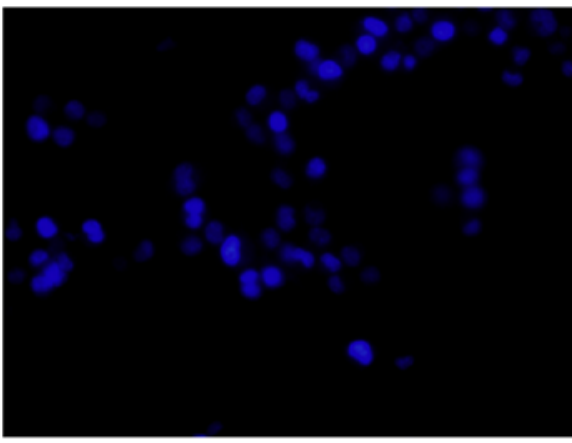

B

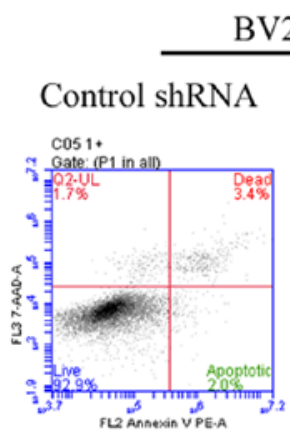

$\mathrm{BV} 2$ cells $+\mathrm{S} 2$

PPM1A ShRNA

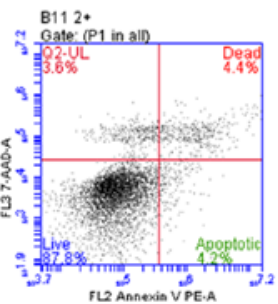

PPMIA ShRNA
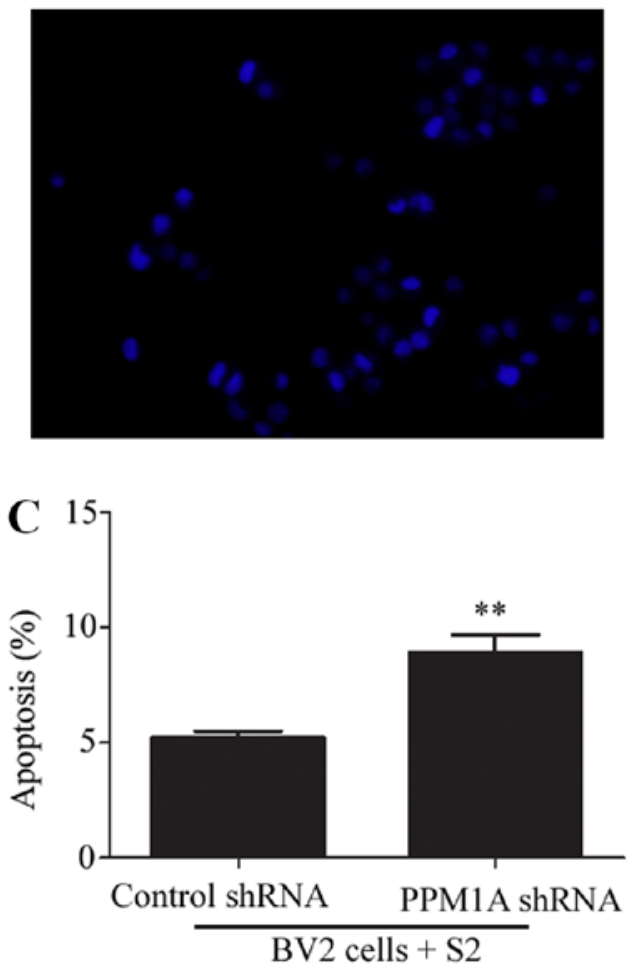

Figure 5. Effects of PPM1A knockdown on apoptosis in BV2 cells infected with B. suis s2. BV2 cells infected with B. suis s2 were transfected with PPM1A shRNA or control shRNA. (A) Cells were stained with Annexin V conjugated to FITC and PI and harvested for a flow cytometry assay. The total apoptosis levels were estimated as the sum of cells in early apoptosis (lower right) and late apoptosis (upper right). Magnification x20. (B) The number of PI/Annexin $\mathrm{V}$ single positive and Annexin V/PI double positive cells was used as a measurement of apoptotic cells $(\mathrm{C})$. Data are expressed as the mean \pm SD. ${ }^{* *} \mathrm{P}<0.01$ vs. control shRNA cells. B. suis, Brucella suis; s2, strain 2; shRNA, short hairpin RNA; PI, propidium iodide.

mycin at $0.2 \mu \mathrm{M}$ had no significant effect on BV2 cell viability compared with DMSO-treated cells (data not shown). Western blot analysis showed that SP100625 appeared to reduce and anisomycin markedly increased the levels of JNK phosphorylation in BV2 cells (Fig. 3A). In comparison to vehicle treated cells, the levels of cleaved caspase-3 were significantly increased in BV2 cells treated with anisomycin, but not altered in those treated with SP100625 (Fig. 3B). To further investigate the effects of JNK signaling modulation on apoptosis in BV2 cells, flow cytometry analysis was performed to detect apoptosis in BV2 cells treated with SP600125 or anisomycin. Treatment with anisomycin significantly increased apoptosis levels in BV2 cells compared with that of the SP600125 treated cells (Fig. 3C). 
Knockdown of PPM1A induces activation of JNK signaling in BV2 cells. To investigate whether PPM1A regulates JNK phosphorylation, the protein levels of JNK and p-JNK were measured using western blot analysis in BV2 cells transfected with PPM1A shRNA. The results indicated that the level of p-JNK in PPM1A shRNA transfected cells was significantly higher than that in control shRNA transfected cells (Fig. 2E), suggesting that PPM1A knockdown activates JNK signaling in BV2 cells.

Knockdown of PPM1A promotes apoptosis in BV2 cells infected with B. suis strain 2. The protein levels of JNK, p-JNK, caspase-3 and cleaved caspase-3 were assessed in BV2 cells infected with $B$. suis strain 2 and transfected with PPM1A shRNA. As shown in Fig. 4, PPM1A shRNA significantly increased the ratios of $\mathrm{p}-\mathrm{JNK} / \mathrm{JNK}$ and the expression levels of cleaved caspase- 3 in BV2 cells infected with $B$. suis strain 2 compared with control cells. Additionally, flow cytometry analysis showed that PPM1A shRNA significantly increased the level of apoptosis in BV2 cells infected with $B$. suis strain 2 compared with cells treated with control shRNA (Fig. 5A and B). Taken together, these data suggest that knockdown of PPM1A promotes apoptosis in BV2 cells infected with $B$. suis strain 2 .

\section{Discussion}

Brucella is an intracellular parasite, which previously, the authors of the current study have reported that the outer membrane protein of Brucella inhibits host cell apoptosis (15), facilitating the replication of bacteria within the cell. In the present study, knockdown of PPM1A promoted apoptosis in BV2 cells infected with B. suis strain 2. Therefore, it is plausible to propose that PPM1A reduces intracellular replication of $B$. suis strain 2 by inducing apoptosis in BV2 cells.

Protein phosphatases are enzymes that catalyze the dephosphorylation of protein molecules in contrast to protein kinases, which phosphorylate proteins. PPM1A is a protein phosphatase, widely present in eukaryotic cells. PPM1A participates in the regulation of the cell cycle, apoptosis, antiviral and other related biological functions (16-18). In the present study, PPM1A was upregulated in BV2 cells infected with B. suis strain 2, and knockdown of PPM1A shown to promote apoptosis. PPM1A may be a potential target to promote the apoptosis of Brucella-infected cells. In addition, JNK was suggested to be a substrate for PPM1A, and increased phosphorylation of JNK can promote apoptosis. These findings indicate that the PPM1A-JNK pathway may be involved in the regulation of BV2 apoptosis after B. suis strain 2 infection. Based on these results, a further study will be focused on the screening of drugs that inhibit PPM1A in vitro and observation of whether these drugs can enhance the efficacy of antibacterial therapy.

Brucellosis is more severe in humans than in domestic animals and causes a variety of clinical symptoms (3), including central nervous system infection related symptoms $(19,20)$. The incidence of brucellosis is higher in areas of intensive agriculture and animal husbandry, including Ningxia, China $(21,22)$.

Brucella is a common zoonotic pathogen that can survive and proliferate within several types of phagocytic and non-phagocytic cells. Phagocytic cells are the main host and Brucella can inhibit apoptosis of these cells (23). In the absence of effective antibiotics treatment, $50 \%$ of brucellosis cases become chronic and cause multiple organ damage, including neurological disorders, bone destruction and cardiovascular damage $(24,25)$. Meningitis is a common cause of fatality in brucellosis $(26,27)$. Improving antibiotic treatment and reducing infection recurrence is the ultimate goal in brucellosis treatment. In the current study, the PPM1A-JNK pathway was revealed to be involved in the regulation of BV2 cell apoptosis after $B$. suis strain 2 infection. Whether PPM1A knockdown can reduce bacterial replication by promoting apoptosis needs to be further explored.

There are several limitations to the present study. Whether only B. suis strain 2-infected BV2 cells undergo apoptosis by PPM1A protein expression knockdown has not been elucidated, as it is difficult to distinguish B. suis strain 2-infected BV2 cells from uninfected cells. Additionally, the similarity between the $B$. suis strain 2 vaccine used in the present study and wild-type Brucella has not been confirmed by sequencing results. This study may inspire the development of new Brucella treatments that promote the apoptosis of host cells, so as to reduce the recurrence of infection.

\section{Acknowledgements}

Not applicable.

\section{Funding}

The current study was supported by grants from the National Natural Science Foundation of China (grant no. 31660030) and the First Class Discipline Construction Project in Colleges and Universities of Ningxia (grant no. NXYLXK2017A05).

\section{Availability of data and materials}

The datasets used and/or analyzed during the current study are available from the corresponding author on reasonable request.

\section{Authors' contributions}

JY, GW, HL and ZW conceived and designed the experiments. JY, GW, and HL conducted all the experiments. WZ and BG contributed to the design of parts of the study and collected and analyzed some of the data. All authors read and approved the final manuscript. All authors read and approved the final manuscript.

\section{Ethics approval and consent to participate}

Not applicable.

\section{Patient consent for publication}

Not applicable.

\section{Competing interests}

The authors declare that they have no competing interests. 


\section{References}

1. Vollmar P, Zange S, Zöller L, Erkel J and Robert Thoma B: Brucellosis, an overview and current aspects. Dtsch Med Wochenschr 141: 1014-1018, 2016 (In German).

2. Zheng R, Xie S, Lu X, Sun L, Zhou Y, Zhang Y and Wang K: A systematic review and meta-analysis of epidemiology and clinical manifestations of human brucellosis in China. Biomed Res Int 2018: 5712920, 2018.

3. Galinska EM and Zagorski J: Brucellosis in humans-etiology, diagnostics, clinical forms. Ann Agric Environ Med 20: 233-238, 2013.

4. Pelerito A, Cordeiro R, Matos R, Santos MA, Soeiro S, Santos J, Manita C, Rio C, Santo M, Paixão E, et al: Human brucellosis in portugal-retrospective analysis of suspected clinical cases of infection from 2009 to 2016. PLoS One 12: e0179667, 2017.

5. Elfaki MG, Alaidan AA and Al-Hokail AA: Host response to brucella infection: Review and future perspective. J Infect Dev Ctries 9: 697-701, 2015.

6. MillerCN, SmithEP,Cundiff JA, Knodler LA, Bailey Blackburn J, Lupashin V and Celli J: A brucella type iv effector targets the cog tethering complex to remodel host secretory traffic and promote intracellular replication. Cell Host Microbe 22: 317-329, 2017.

7. Carvalho TF, Haddad JP, Paixão TA and Santos RL: Meta-Analysis and advancement of brucellosis vaccinology. PLoS One 11: e0166582, 2016.

8. Schaaf K, Smith SR, Duverger A, Wagner F, Wolschendorf F, Westfall AO, Kutsch O and Sun J: Mycobacterium tuberculosis exploits the PPM1A signaling pathway to block host macrophage apoptosis. Sci Rep 7: 42101, 2017.

9. Sun J, Schaaf K, Duverger A, Wolschendorf F, Speer A, Wagner F, Niederweis $M$ and Kutsch O: Protein phosphatase, $\mathrm{Mg} 2+/ \mathrm{Mn} 2+-$ dependent $1 \mathrm{~A}$ controls the innate antiviral and antibacterial response of macrophages during HIV-1 and mycobacterium tuberculosis infection. Oncotarget 7: 15394-15409, 2016.

10. Xiang W, Zhang Q, Lin X, Wu S, Zhou Y, Meng F, Fan Y, Shen T, Xiao M, Xia Z, et al: PPM1A silences cytosolic RNA sensing and antiviral defense through direct dephosphorylation of MAVS and TBK1. Sci Adv 2: e1501889, 2016.

11. Siqueira MDS, Ribeiro RM and Travassos LH: Autophagy and its interaction with intracellular bacterial pathogens. Front Immunol 9: 935, 2018.

12. Li Y, Wei C, Xu H, Jia J, Wei Z, Guo R, Jia Y, Wu Y, Li Y, Qi X, et al: The immunoregulation of Th17 in host against intracellular bacterial infection. Mediators Inflamm 19: 6587296, 2018.

13. Heppner FL, Ransohoff RM and Becher B: Immune attack: The role of inflammation in alzheimer disease. Nat Rev Neurosci 16 : 358-372, 2015.
14. Yang J, Zhang J, Xu T, Wang Y and Wang Z: Establishment of $\mathrm{BV} 2$ cell line with steady knockdown of $\mathrm{Mg}^{2+} / \mathrm{Mn}^{2+}$-dependent protein phosphatase 1A(PPM1A). Xi Bao Yu Fen Zi Mian Yi Xue Za Zhi 34: 818-823, 2018 (In Chinese).

15. Ma QL, Liu AC, Ma XJ, Wang YB, Hou YT and Wang ZH: Brucella outer membrane protein Omp25 induces microglial cells in vitro to secrete inflammatory cytokines and inhibit apoptosis. Int J Clin Exp Med 8: 17530-17535, 2015.

16. Smith SR, Schaaf K, Rajabalee N, Wagner F, Duverger A, Kutsch $\mathrm{O}$ and Sun J: The phosphatase PPM1A controls monocyte-to-macrophage differentiation. Sci Rep 8: 902, 2018.

17. Wang Y, Dow EC, Liang YY, Ramakrishnan R, Liu H, Sung TL, Lin $X$ and Rice AP: Phosphatase PPM1A regulates phosphorylation of thr-186 in the Cdk9 T-loop. J Biol Chem 283: 33578-22584, 2008.

18. Sun W, Yu Y, Dotti G, Shen T, Tan X, Savoldo B, Pass AK, Chu M, Zhang D, Lu X, et al: PPM1A and PPM1B act as IKKbeta phosphatases to terminate TNFalpha-induced IKKbeta-NF-kappaB activation. Cell Signal 21: 95-102, 2009.

19. Levy J, Shneck M, Marcus M and Lifshitz T: Brucella meningitis and papilledema in a child. Eur J Ophthalmol 15: 818-820, 2005

20. Haji-Abdolbagi M, Rasooli-Nejad M, Jafari S, Hasibi M and Soudbakhsh A: Clinical and laboratory findings in neurobrucellosis: Review of 31 cases. Arch Iran Med 11: 21-25, 2008.

21. Li YJ, Li XL, Liang S, Fang LQ and Cao WC: Epidemiological features and risk factors associated with the spatial and temporal distribution of human brucellosis in China. BMC Infect Dis 13: $547,2013$.

22. Lai S, Zhou H, Xiong W, Gilbert M, Huang Z, Yu J, Yin W, Wang L, Chen Q, Li Y, et al: Changing epidemiology of human brucellosis, China, 1955-2014. Emerg Infect Dis 23: 184-194, 2017.

23. Deng Y, Liu X, Duan K and Peng Q: Research progress on brucellosis. Curr Med Chem 26: 5598-5608, 2018.

24. Tu L, Liu X, Gu W, Wang Z, Zhang E, Kahar A, Chu G and Zhao J: Imaging-assisted diagnosis and characteristics of suspected spinal brucellosis: A retrospective study of 72 cases. Med Sci Monit 24: 2647-2654, 2018.

25. Sabzi F and Faraji R: Brucella pericarditis: A forgotten cause of chest pain. Caspian J Intern Med 8: 116-118, 2017.

26. Olsen SC and Palmer MV: Advancement of knowledge of brucella over the past 50 years. Vet Pathol 51: 1076-1089, 2014

27. Moreno E and Moriyon I: Brucella melitensis: A nasty bug with hidden credentials for virulence. Proc Natl Acad Sci USA 99: $1-3,2002$.

(i) $\ominus$ This work is licensed under a Creative Commons

Attribution-NonCommercial-NoDerivatives 4.0 International (CC BY-NC-ND 4.0) License. 